\title{
Comparison and Confirmation of Quantitative Trait Loci Conferring Partial Resistance to Rice Sheath Blight on Chromosome 9
}

\author{
S. M. Zuo, Y. J. Zhu, Y. J. Yin, H. Wang, Y. F. Zhang, Z. X. Chen, S. L. Gu, and X. B. Pan, Key Lab for Crop Genetics and Physiol- \\ ogy of Jiangsu Province and Key Lab of Plant Functional Genomics, Ministry of Education, Yangzhou University, Yangzhou 225009, \\ P.R. China
}

\begin{abstract}
Zuo, S. M., Zhu, Y. J., Yin, Y. J., Wang, H., Zhang, Y. F., Chen, Z. X., Gu, S. L., and Pan, X. B. 2014. Comparison and confirmation of quantitative trait loci conferring partial resistance to rice sheath blight on chromosome 9. Plant Dis. 98:957-964.

Sheath blight (SB), caused by Rhizoctonia solani, is one of the worst rice (Orzya sativa) diseases worldwide. Resistance to the SB disease in rice is a complex trait controlled by quantitative trait loci (QTLs). Through map integration, we found several previously identified SB resistance (SBR) QTLs reported in inconsistent regions on the long arm of chromosome 9. Five of them were detected on 'Jasmine 85' (J85), 'Minghui 63' (MH63), and 'Lemont' (LMNT) rice and were designated $q S B-9^{185}-1, q S B-9^{J 85}-2, q S B-9^{M H 63}-1, q S B-9^{M H 63}-2$, and $q S B$ $9^{L M N T}$, respectively, in the present study. To further verify and physically map the five potential SBR QTLs, we introduced these SBR QTLs into a common susceptible variety (LMNT) and developed a few chromosomal segment substitution lines through marker-assisted selec-

tion. After artificial inoculation with the SB fungus, we were able to validate $q S B-9^{\prime 85}-2$ but not the other four SBR QTLs; whereas, on MH63, an SBR QTL designated $q S B-9^{M H 63}-3$ was confirmed in the region defined by markers Y 83 and Y91.8 that included $q S B-9^{185}-2$, covering approximately $1,235 \mathrm{~kb}$. Both $q S B-9^{J 85}-2$ and $q S B-9^{M H 63}-3$ appeared to be dominant resistance genes and contributed to similar levels to SB resistance, reducing SB disease severity by approximately 1.0 on a 0 -to-9 SB disease rating system. After comparing with another confirmed SBR QTL $\left(q S B-9^{T Q}\right)$ from 'Teqing' rice (TQ), we conclude that $q S B-9^{J 85}-2, q S B-9^{M H 63}-3$, and $q S B-9^{T Q}$ are probably controlled by the same allelic resistance genes. These results will accelerate the utilization of this major SBR QTL and its map-based cloning.
\end{abstract}

Rice sheath blight (SB) disease, caused by the soilborne fungal pathogen Rhizoctonia solani Kühn AG1-1A, is a major disease of rice worldwide. Under highly intensive rice production systems, rice SB disease can cause yield losses up to 50\% (23). With the wide deployment of semidwarf rice varieties and the excessive use of fertilizers, the SB disease has become more and more serious in recent years. For instance, in south China, the SB disease has become the most severe disease in rice, which caused yield losses up to 1.97 times of that caused by rice blast disease (38). The necrotrophic SB fungus has a broad range of hosts, infecting both monocots and dicots, making it difficult to control this disease in field management through crop rotation (23). Due to the high cost of fungicide application, the development and use of SB-resistant rice cultivars is considered the most economically effective and environmentally friendly method of disease control.

To date, however, none of the rice genotypes screened are immune to SB, although substantial differences in their resistance levels have been widely observed $(2,17,23,38)$. With the development of molecular markers and the advancement in quantitative genetics, the differences in SB resistance levels have been attributed to quantitative trait loci (QTLs; 4,8,10,12,20,32). Thus far, over $50 \mathrm{SB}$ resistance (SBR) QTLs have been reported in different rice genomes $(13,23,38)$. These SBR QTLs were detected from different rice genotypes, including wild accessions and deep-water rice varieties $(3,4,38)$. Unfortunately, most of these SBR QTLs were just preliminarily mapped, lacking further validation, and many inconsistencies existed among different studies or years (38).

Corresponding author: S. M. Zuo,

E-mail: smzuo@yzu.edu.cn and xbpan@yzu.edu.cn

S. M. Zuo and Y. J. Zhu contributed equally to this research.

Accepted for publication 24 January 2014.

http://dx.doi.org/10.1094/PDIS-09-13-0940-RE

(C) 2014 The American Phytopathological Society
Some SBR QTLs were co-localized with QTLs for morphological attributes, especially plant height, or for developmental traits such as heading date $(6,23,38)$. The uncertainty of these SBR QTLs has limited their application in breeding to introduce these SBR QTLs into elite rice cultivars for improving their SB resistance by marker-assisted selection (MAS; 13,18,38).

By using different parents and mapping populations, the long arm of rice chromosome 9 was found to host several potential SBR QTLs. The donors for these SBR QTLs included 'Jasmine 85' (J85), 'Minghui 63' (MH63), and 'Teqing' (TQ) (5,10,13,18,20, 27,32). Because the mapping regions for these SBR QTLs were not all consistent among different studies, in this study we renamed them as $q S B-9^{T Q}, q S B-9^{J 85}$, and $q S B-9^{M H 63}$ based on their donor parents. The existence of $q S B-9^{T Q}$ has been validated in several independent studies by using different mapping populations derived from the same parental cross of TQ $\times$ LMNT, such as $\mathrm{F}_{3-4}$ progeny (10), $\mathrm{F}_{2}$ clonal lines (18), $\mathrm{BC}_{3} \mathrm{~F}_{1-2}$ backcross lines (27), recombinant inbred lines (RILs; 20), and introgression lines (28). Overall, in these studies, the $q S B-9^{T Q}$ was located in very similar regions. However, for $q S B-9^{J 85}$, two independent studies produced completely different mapping regions $(12,13,32)$. Similarly, inconsistent regions were reported for $q S B-9^{M H 63}$ (5). Because different genetic backgrounds can significantly affect gene expression, leading to phenotypic impacts, QTL-mapping studies based on RIL progeny and randomly segregating $\mathrm{F}_{2}$ and $\mathrm{F}_{3-4}$ families may not accurately define the position of a QTL (29).

In this study, we integrated the above potential SBR QTLs identified on the long arm of chromosome 9 onto the rice physical map and noted that their mapped regions either completely coincided or overlapped. In order to validate these SBR QTLs and investigate whether they are independent SBR QTLs, we adopted MAS to introduce them into a common SB-susceptible parent, LMNT, for generating chromosomal segment substitution lines (CSSLs). Compared with the above-mentioned randomly segregating populations, CSSLs in the same genetic background are better suited for verifying a QTL and determining its genetic effects and location $(1,26)$. Through artificial SB inoculation, we evaluated the resistance performance of different CSSLs to the SB disease. After 
comparing the genotype with the phenotype of each line, we validated the presence and determined the location of some of these SBR QTLs onto the bottom of chromosome 9. Their resistance effects in reducing the SB disease severity score were estimated. Results in this study will accelerate the utilization of this major SBR QTL in breeding program by MAS and facilitate its mapbased cloning.

\section{Materials and Methods}

Plant materials. Rice (Oryza sativa L.) variety 'Lemont' (LMNT), a tropical japonica cultivar, was obtained from Louisiana State University. U.S. J85 and MH63 are indica rice cultivars, obtained from the International Rice Research Institute, Philippines, and Fujian, China, respectively. Among them, LMNT is most susceptible to the SB disease, while J85, MH63, and 'Teqing' (TQ) are all moderately resistant $(5,12,13,32)$.

SBR QTL interval integration and development of molecular markers. Sequences of the molecular markers flanking the SBR QTLs mapped at the long arm of chromosome 9 were obtained from Gramene (www.gramene.org). The physical position of each marker was anchored on a sequence map of the japonica rice 'Nipponbare' in order to construct an integrated map, using the basic local alignment search tool (BLASTn) provided in Gramene.

Public simple sequence repeat (SSR) markers located in the integrated map were employed first to perform polymorphic analysis between the susceptible recurrent parent LMNT and each of donor parents, MH63, and J85. Polymorphic SSR markers were kept for subsequent experiments. When no polymorphic SSR markers were found, the insertion and deletion (InDel) markers were developed according to the procedure as follows. The Nipponbare sequence that included the InDel sequence divergence between japonica Nipponbare and indica '93-11', revealed by the BLASTn program in the National Center for Biotechnology Information (http:// blast.ncbi.nlm.nih.gov/), were downloaded, and the primers were designed on both sides of candidate InDel polymorphisms using the Primer Premier 5.0 software (http://www.tucows.com/preview/ 205452) for developing candidate InDel markers (33). Candidate InDel markers were validated for polymorphism between the parents.

Polymerase chain reaction. Plant genomic DNA was isolated as described by Murray and Thompson (15). Polymerase chain reaction $(\mathrm{PCR})$ mixture $(20 \mu \mathrm{l})$ contained $2.0 \mu \mathrm{l}$ of $\mathrm{MgCl}_{2}(25$ $\mathrm{mM}), 10 \times$ PCR buffer, $3.084 \mathrm{mM}$ primers, $0.4 \mu \mathrm{l}$ of dNTP $(10$ $\mathrm{mM}), 0.2 \mu \mathrm{l}$ of Taq DNA polymerase $(5 \mathrm{U} / \mu \mathrm{l})$, and $2.0 \mu \mathrm{l}$ of DNA template (100 to $150 \mu \mathrm{g} / \mathrm{ml})$. The PCR conditions were as follows: predenaturing at $94^{\circ} \mathrm{C}$ for $5 \mathrm{~min} ; 35$ cycles at $94^{\circ} \mathrm{C}$ for $1 \mathrm{~min}, 55^{\circ} \mathrm{C}$ for $1 \mathrm{~min}$, and $72^{\circ} \mathrm{C}$ for $1 \mathrm{~min}$; and a final extension at $72^{\circ} \mathrm{C}$ for 10 min. The samples were then placed on ice. PCR products were separated by electrophoresis in a 3 to $4 \%$ agarose gel for those markers with obvious polymorphism or in a $6 \%$ polyacrylamide gel for weak polymorphic markers (7).

Development of populations for validating SBR QTLs. Susceptible LMNT was used as the recurrent recipient parent to cross and backcross with the donor parents J85 and MH63, respectively. From the $\mathrm{BC}_{2} \mathrm{~F}_{1}$ generation, the polymorphic markers located in the integrated SBR QTL interval were used to select for a donor chromosomal segment, which was designed as foreground (forward) selection. Through foreground selection by markers, the genotypes (heterozygote or homozygote) of each plant on the chromosomal regions of interest could be easily determined before transplanting. From the $\mathrm{BC}_{4} \mathrm{~F}_{1}$ generation on, in addition to foreground selection, we performed background selection to monitor genetic background of each selected plant. The background selection was conducted by 70 polymorphic markers that were relatively evenly distributed on the remaining chromosomal regions (data not shown). After both selections, we selected a few $\mathrm{BC}_{4} \mathrm{~F}_{1}$ plants that harbored various lengths of donor chromosomal segments and shared the same genetic background with the recurrent parent $\mathrm{LMNT}$ at 70 marker loci. Each of the selected $\mathrm{BC}_{4} \mathrm{~F}_{1}$ plants was then used to further backcross with LMNT to generate more than 100 hybrid seeds $\left(\mathrm{BC}_{5} \mathrm{~F}_{1}\right)$. In each $\mathrm{BC}_{5} \mathrm{~F}_{1}$ population, the plants that showed the heterozygous genotype at the regions replaced by the donor chromosomal segments were selected by the foreground selection. Then, among these selected plants, those that showed homozygous genotypes at the remainder of the region determined by background selection were collected for SB-fungus inoculation. We defined these populations as heterozygous CSSL lines (hCSSLs), and they were used to investigate the effect of SBR QTLs in the heterozygous condition.

One individual of each $\mathrm{BC}_{5} \mathrm{~F}_{1}$ population was then selected for self-pollination to produce homozygous donor genotypes at the substitution region in the $\mathrm{BC}_{5} \mathrm{~F}_{2}$ generation. The progeny $\left(\mathrm{BC}_{5} \mathrm{~F}_{3}\right)$ of each homozygous plant was named a CSSL. The CSSLs developed were used to further validate the SBR QTLs.

In order to analyze the allelic relationship among the confirmed SBR QTLs on chromosome 9 from different donors (J85, MH63, and TQ), we developed three hybrid lines (HLs) in susceptible LMNT background. The first HL was designated NILqSB9 ${ }^{\mathrm{MH} 63 / \mathrm{J} 85}$, and was obtained by crossing near-isogenic lines (NILs) CSSL-E-qSB9 ${ }^{\mathrm{MH} 63}$ and CSSL-E-qSB9 ${ }^{\mathrm{J} 85}$. Another two HLs, named NIL-qSB9 ${ }^{\mathrm{MH} 63 / \mathrm{TQ}}$ and NIL-qSB9 ${ }^{\mathrm{I85} / \mathrm{TQ}}$, were developed by crossing CSSL-E-qSB9 ${ }^{\mathrm{MH} 63}$ and NIL-qSB9 ${ }^{\mathrm{TQ}}$, and CSSLE-qSB $9^{\mathrm{J} 85}$ and NIL-qSB9 ${ }^{\mathrm{TQ}}$, respectively. NIL-qSB9 ${ }^{\mathrm{TQ}}$ was an NIL of LMNT that contained $q S B-9^{T Q}$ in LMNT background and showed significantly higher resistance to SB disease than that of LMNT (30). Each HL contained at least 60 plants.

Measurement of morphological and developmental traits. Seven traits were assessed on all CSSLs: heading date (HD), plant height (PH), flag-leaf height (FLH), flag-leaf length (FLL), panicle length (PL), tiller number (TN), and tiller angle (TA). All traits, except HD, were measured from 10 plants in each plot. HD was recorded as days from sowing to the date when panicles emerged for $50 \%$ of the plants in a plot. PH and FLH were measured from the soil surface to the tips of the tallest panicles $(\mathrm{PH})$ and flag leaves (FLH). FLL was measured as the length of the flag leaves of three main tillers of each plant before maturity. PL was measured after maturity. TN was counted as the number of tillers of each plant before maturity. TA was measured as the maximum degree among tillers in the plant using a protractor.

Field experiment design. Trials were carried out on the experimental farm at Yangzhou University. For the field dedicated to SB inoculation, the water level was maintained at least $3.0 \mathrm{~cm}$ above the ground until SB disease scoring. All materials were sown on 8 May and transplanted on 8 June with a completely randomized design in the rice field. The distances between rows and between plants in the row were 35 and $20 \mathrm{~cm}$, respectively.

For hCSSLs, each line contained a different number of plants due to the different numbers of hybrid seed $\left(\mathrm{BC}_{5} \mathrm{~F}_{1}\right)$ obtained. In total, the number of plants in each of the hCSSLs was 11 to 57; each plot contained two to six rows with 10 plants/row in the field. In all, 12 plants were grown in each row, including 1 LMNT plant on each end and 10 hCSSLs plants in between the LMNT plants. For CSSLs and HLs, including the controls, each line was grown in five plots with five replications in the field, and each plot comprised three rows with 12 plants/row. In the HL plot, either side of the middle row was transplanted with LMNT plants because of the limited number of HL plants.

In order to maintain a consistent, favorable microenvironment for SB fungus infection and disease development, susceptible LMNT plants were grown as barrier rows on all four borders of each experimental block. The border region contained 10 LMNT plants per row and was separated from the experimental block by a 1.0-m wide walkway.

SB fungus inoculation and disease scoring. We used $R h i$ zoctonia solani strain YN-7 (the original name was RH-9) with moderate pathogenicity, obtained from the Plant Protection Institute of Jiangsu Academy of Agriculture, for the inoculation experiments (33). Woody matchsticks were used as the substrate. Autoclaved, truncated thin matchsticks with the length of 0.8 to $1.0 \mathrm{~cm}$ were incubated with the $\mathrm{YN}-7$ strain on potato dextrose broth 
(PDB) medium for 3 to 5 days at room temperature (16). The depth of the PDB medium was equal to the matchsticks to ensure fully contact of aerial hyphae and matchsticks. Once the fungus had spread over the surface of the culture plate and each matchstick was sufficiently covered with aerial hyphae of $R$. solani, the matchsticks were ready for use as the inoculum $(16,32)$.

The inoculation was done at the late tillering stage. In order to ensure successful inoculation, the main stem and the largest two tillers of each plant were inoculated $(16,32)$. Using a nipper, the inoculum was placed inside the third sheath from the top without changing the sheath-stem holding status. Because the second sheath from top of the plant at this stage does not elongate further, there was minimal chance of the YN-7-infested toothpick falling out of the leaf sheath. Furthermore, the microenvironment within the leaf sheath was optimal for SB fungus infection, leading to consistent successful inoculation. $(16,24,32)$.

Disease severity was rated approximately 30 days after heading, when disease severity was at a maximum because conditions in both the plant and the climate in Yangzhou for were unfavorable for further disease development. A modified 0-to-9 disease scoring system proposed by Rush et al. (22) was adopted in this study, where 0 was defined as no disease and 9 indicates that the plant was dead or collapsed (39). This modified rating scale is capable of measuring individual tillers on a plant with improved objectivity (33).

Data analysis. The SB disease severity rating data of each population was processed by the analysis of variance (ANOVA) program packaged in Matlab 7.4.0 (license number 318060). The completely randomized model with single factor (rice lines) in ANOVA was used to test the statistical difference in SB disease severity among hCSSLs, among CSSLs, and among HLs, and to test the statistical difference in agronomic traits among CSSLs and the LMNT control. The disease score of each plant in hCSSLs was used in ANOVA, while the average disease score of 10 plants from each replication in CSSLs was used in ANOVA. Least significant differences were used to compare the lines that differed in their SB disease ratings.

\section{Results}

An integrated map for the SBR QTLs located at the lower part of chromosome 9. By using markers flanking the QTLs, we integrated the potential SBR QTLs at the lower part of chromosome 9 onto the rice physical map (Fig. 1). The integrated longest interval for these potential SBR QTLs was from markers RM409 to RG451, covering approximately $8 \mathrm{Mb}$ (Fig. 1). From the inte-

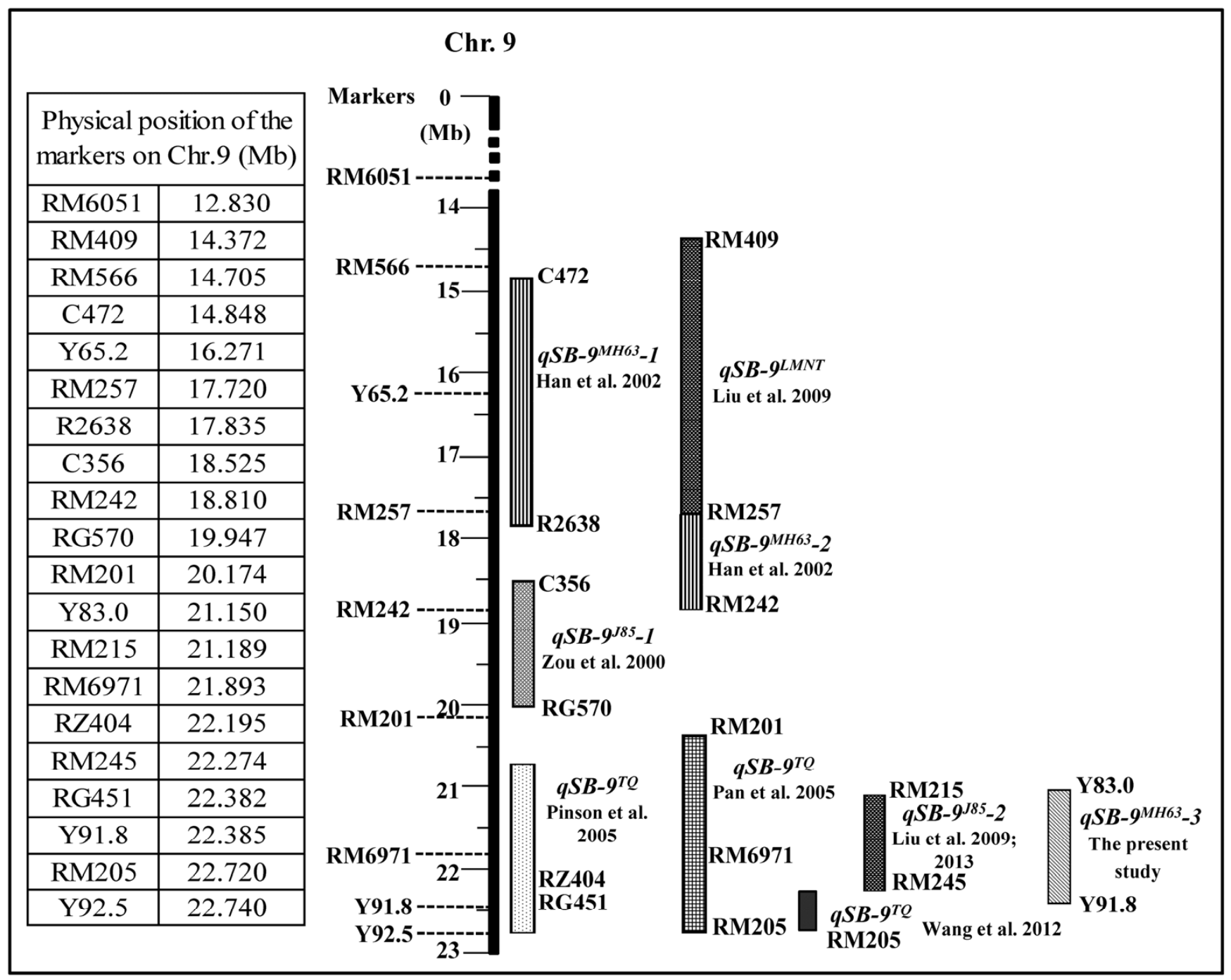

Fig. 1. Integrated map for the sheath blight resistance quantitative trait loci (SBR QTLs) located at the lower part of chromosome 9. Flanking markers for each SBR QTL from the corresponding study are anchored on the sequence map of 'Nipponbare' rice for drawing the integrated map. Physical positions of the markers on chromosome 9 are listed in the table on the left panel. qSB-9J85-1, qSB-9J85-2, qSB-9TQ, qSB-9MH63-1, qSB-9MH63-2 and qSB-9LMNT are renamed in the present study based on their marker interval and the donor parent of the resistant alleles underlying each SBR QTL (i.e., Jasmine 85 [J85], Teqing [TQ], Minghui 63 [MH63], and Lemont [LMNT]). qSB-9MH63-3 is a SBR QTL that was first identified on MH63 and physically mapped in the present study. Molecular markers on the left side of the schematic chromosome are the markers showing polymorphisms between the recurrent parent LMNT and each of the donor parents (J85, TQ, and MH63). In this study, they are used in the MAS program for constructing chromosomal segment substitution lines that harbor various lengths of donor chromosomal segments. 
grated map, we found that the mapping regions of some SBR QTLs were inconsistent among different studies, except for $q S B$ $9^{T Q}$. In order to distinguish these SBR QTLs in different regions, we renamed them as $q S B-9^{J 85}-1, q S B-9^{J 85}-2, q S B-9^{M H 63}-1, q S B$ $9^{M H 63}-2$, and $q S B-9^{L M N T}$. To our best knowledge, among all SBR QTLs on this integrated map, only $q S B-9^{T Q}$ has been confirmed $(28,30,37)$.

In order to validate these SBR QTLs and compare their physical positions, we developed 10 markers (Table 1) that showed polymorphisms between the recurrent parent LMNT and each of the donor parents, J85 and MH63, to construct CSSLs. Markers RM6051 and Y92.5, which are located near the two ends of the integrated longest interval, were used to monitor the genetic background. In order to increase the efficacy of MAS, we subsequently developed markers RM566 and Y91.8 to replace restriction fragment length polymorphism markers $\mathrm{C} 472$ and RG451, respectively. RM566 is closest to C472 and Y91.8 closest to RG451 (Fig. 1).

Development and characteristics of chromosomal segment substitution lines. Through MAS, we introduced the abovedescribed SBR QTLs into the common susceptible LMNT. In $\mathrm{BC}_{4} \mathrm{~F}_{1}$ populations, we obtained 10 plants from each backcross after both foreground and background selections. These plants possessed almost the same genetic background as the recurrent parent LMNT, which was verified by screening with 70 polymorphic markers; but they had acquired various lengths of donor chromosomal segments in the integrated interval. In the backcross progeny $\left(\mathrm{BC}_{5} \mathrm{~F}_{1}\right)$ of each of these $\mathrm{BC}_{4} \mathrm{~F}_{1}$ plants, we obtained 10 types of hCSSLs (Fig. 2). Except for hCSSLs A and B, the remaining eight hCSSLs contained partial donor chromosomal segments in various lengths that resulted from crossovers within the interval. When staggered together, these partial donor chromosomal segments covered the whole integrated interval (Fig. 2). By visual observation, we did not find any difference in morphological or developmental traits among the $10 \mathrm{hCSSLs}$ and the LMNT control, apart from TA. The plants in hCSSLs B, C, F, G, and $\mathrm{K}$ all showed apparently larger TAs than that of the other lines and the LMNT control.

One individual from each of hCSSLs was then used to produce a homozygous line (CSSL) at all markers in the integrated longest region (Fig. 2), resulting in 10 CSSLs for each backcross. We recorded some of the morphological and developmental traits that were considered to be associated with SB resistance for these CSSLs (Table 2). The results showed that, except for TA, all CSSLs shared the same morphological traits with the control LMNT, consistent with the results of visual observation on hCSSLs. With regard to TA, significant differences were found between CSSLs B, C, F, G, and K, and CSSLs A, D, E, H, and I (Table 2). In addition to these morphological traits, we found that all CSSLs exhibited developmental patterns similar to LMNT based on visual observation from the seeding stage to the mature stage in

Table 1. Sequences of polymorphic molecular markers developed or used in the present study

\begin{tabular}{lll}
\hline Marker $\mathbf{n a m e}^{\mathbf{z}}$ & Forward sequence $\left(\mathbf{5}^{\prime} \mathbf{-} \mathbf{3}^{\prime}\right)$ & Reverse sequence $\left(\mathbf{5}^{\prime}-\mathbf{3}^{\prime}\right)$ \\
\hline RM566 & ACCCAACTACGATCAGCTCG & CTCCAGGAACACGCTCTTTC \\
RM6501 & AGGCTGATCCAAGATCCATG & CCCGGAGGCTGATTCTTG \\
RM257 & CAGTTCCGAGCAAGAGTACTC & GGATCGGACGTGGCATATG \\
RM242 & GGCCAACGTGTGTATGTCTC & TATATGCCAAGACGGATGGG \\
RM201 & CTCGTTTATTACCTACAGTACC & CTACCTCCTTCTAGACCGATA \\
RM6971 & TTTGCGAACTAGACAAGGCC & TTATGCCTTCGCTAATGG \\
Y91.8 & ACCTTGGCTGGTGTCTTC & ACACCGACCAAAGTCAAAT \\
Y92.5 & TAAAATGTAGGACACCAAATC & CCTGAGTCCCTCTTAAACCAAA \\
Y80.3 & TCAATAACCCTCCTCTTCATCCT & GACTCCGGACTAGACCAATC \\
\hline
\end{tabular}

${ }^{\mathrm{z}}$ Markers beginning with 'RM' were public simple-sequence repeat markers that were retrieved from the Gramene database (www.gramene.org) and markers beginning with ' $\mathrm{Y}$ ' were insertion and deletion markers that were developed in the present study.

Markers
Chr.9
Interval distan
CSSLs

CK-Lemont
A-MH63 / A-J85
B-MH63 / B-J85
E-MH63 / E-J85
C-MH63 / C-J85
K-MH63 / C-J85
G-MH63 / C-J85
F-MH63 / F-J85
D-MH63 / D-J85
I-MH63 / I-J85
H-MH63 / H-J85

(Kb)
Phen-
otype
S
S
R
R
R
R
R
R
S
S
S

$\begin{array}{lllll}\text { RM6051 } & \text { RM566 } & \text { Y65.2 } & \text { RM257 } & \text { RM242 }\end{array}$

RM201 Y83.0 RM6971 Y91.8 Y92.5
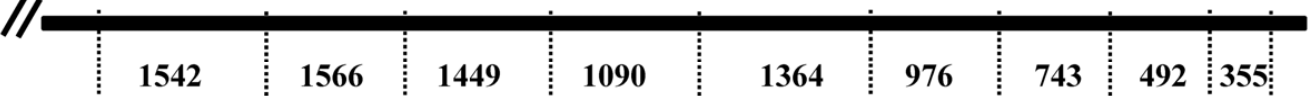
the field. Taken together, we confirmed that the CSSLs had almost the same genetic background as LMNT; this information is critical to the accurate evaluation of the effects of these SBR QTLs.

Validation and physical mapping of SBR QTLs on chromosome 9 of MH63 and J85. Because hCSSL A and CSSL A contained only the recipient chromosomal DNA in the integrated interval, they were regarded as the potential susceptible control in addition to LMNT. In contrast, hCSSL B and CSSL B, which possessed a resistant donor chromosomal segment covering the whole integrated interval, were regarded as the potential relatively resistant control.

The ANOVA and multiple comparisons were used to analyze possible statistically significant differences in the SB disease ratings among the hCSSLs and control (Tables 3 and 4). The results showed that, in both backcrosses, highly significant differences were found among hCSSLs (Table 3). Results of multi-comparisons (Table 4) showed that, in each backcross, the hCSSLs could be easily divided into two groups with statistically significant differences in their SB disease ratings: group one included hCSSLs C, $\mathrm{E}, \mathrm{F}, \mathrm{G}, \mathrm{K}$, and the resistant control hCSSL B that was regarded as the relatively resistant phenotype; group two consisted of the remaining three hCSSLs and the susceptible phenotype control hCSSL A. These data imply that the overlapping donor chromosomal segment in the resistant hCSSLs is responsible for the SB resistance phenotype.

To further confirm the contribution of this defined chromosomal segment, the corresponding homozygous CSSLs were developed. The significant differences in resistance among these CSSLs to the SB disease were revealed after the ANOVA analysis (Tables 5 and 6). Similar to the results obtained from hCSSLs, the CSSLs in each backcross could be divided into two types of phenotypes: susceptible and relatively resistant (Table 6). The relatively resistant group consisted of CSSLs B, C, E, F, G, and K, which had lower disease ratings than that of the susceptible group, which contained CSSLs $\mathrm{A}, \mathrm{D}, \mathrm{H}$, and $\mathrm{M}$ (Table 6). These results were identical to those obtained from using the hCSSLs. In summary, the results confirmed that the overlapping donor chromosomal segment in the resistant CSSLs confers resistance to SB.

By comparing the genotype and phenotype of each of the hCSSLs and CSSLs, we found that marker RM6971 co-segregated with the phenotype in the present populations. Moreover, we found that lines $\mathrm{C}, \mathrm{F}$, and $\mathrm{K}$ had crossovers between RM6971 and its closest right-side marker, Y91.8 (Fig. 2). These lines showed the resistant phenotype but had genotype of marker Y91.8 that was present in susceptible lines. Therefore, we concluded that SBR QTLs on the long arm of chromosome 9 from both MH63 and J85 resided on the left side of Y91.8. Similarly, we found that line E had a crossover between RM6971 and its closest left marker, RM201. This indicated that both SBR QTLs located at the right side of RM201. Because lines hCSSL E and CSSL E all showed normal TA like that of LMNT (Table 2), we determined that both SBR QTLs located at the right side of the TAC1 locus. We then developed one more polymorphic marker, Y83, at the right side of TAC1. Genotype analysis showed that both hCSSL E and CSSL E had susceptible LMNT genotype at the Y83 marker (Fig. 2). Consequently, the Y83 marker was regarded as the closest left-side marker of the SBR QTLs. Taken together, we conclude that SBR QTLs on chromosome 9 from MH63 and J85 are both located in the region between markers Y83 and Y91.8. This region covers approximately $1,235 \mathrm{~kb}$ on the rice reference sequence map (Fig.

Table 4. Multi-comparisons of sheath blight scores for 10 heterozygous chromosome segment substitution lines (hCSSLs) from each of two backcrosses $^{\mathrm{z}}$

\begin{tabular}{lccccc}
\hline & \multicolumn{4}{c}{ hCSSLs (2006) } \\
\cline { 2 - 3 } & \multicolumn{3}{c}{ Jasmine 85/Lemont } & & \multicolumn{2}{c}{ Minghui 63/Lemont } \\
\cline { 2 - 3 } \cline { 5 - 6 } Types & $\boldsymbol{N}$ & DS & & \multicolumn{1}{c}{$\boldsymbol{N}$} & DS \\
\hline hCSSL E & 11 & $7.20 \mathrm{~B}$ & & 22 & $7.14 \mathrm{~B}$ \\
hCSSL C & 52 & $7.03 \mathrm{~B}$ & & 52 & $7.13 \mathrm{~B}$ \\
hCSSL K & 40 & $7.20 \mathrm{~B}$ & & 45 & $7.19 \mathrm{~B}$ \\
hCSSL G & 40 & $7.19 \mathrm{~B}$ & & 47 & $6.93 \mathrm{~B}$ \\
hCSSL F & 44 & $7.14 \mathrm{~B}$ & & 49 & $7.19 \mathrm{~B}$ \\
hCSSL D & 45 & $8.16 \mathrm{~A}$ & & 46 & $8.20 \mathrm{~A}$ \\
hCSSL M & 36 & $8.03 \mathrm{~A}$ & & 40 & $8.05 \mathrm{~A}$ \\
hCSSL H & 46 & $8.08 \mathrm{~A}$ & & 53 & $7.88 \mathrm{~A}$ \\
hCSSL A & 57 & $8.13 \mathrm{~A}$ & & 51 & $8.15 \mathrm{~A}$ \\
hCSSL B & 45 & $7.21 \mathrm{~B}$ & & 56 & $7.01 \mathrm{~B}$ \\
\hline
\end{tabular}

${ }^{\mathrm{z}} N=$ number of plants and DS $=$ disease scores. Values with different capital letters indicate the difference on $1 \%$ level of statistic significance $(P<0.01)$ while values with same letter indicate not statistically significant $(P>0.05)$.

Table 2. Multi-comparisons of morphological and developmental traits among the recurrent parent Lemont and 10 chromosome segment substitution lines $(\mathrm{CSSLs})^{\mathrm{z}}$

\begin{tabular}{|c|c|c|c|c|c|c|}
\hline Lines & HD (day) & PH (cm) & PL (cm) & FLL (cm) & TN (number) & TA $\left({ }^{\circ} \mathbf{C}\right)$ \\
\hline Lemont & $99.33 \mathrm{a}$ & $78.81 \mathrm{a}$ & $22.37 \mathrm{a}$ & $29.31 \mathrm{a}$ & $6.14 \mathrm{a}$ & $29.11 \mathrm{~A}$ \\
\hline CSSL A & $98.00 \mathrm{a}$ & $79.24 \mathrm{a}$ & $20.96 \mathrm{a}$ & $30.03 \mathrm{a}$ & $6.73 \mathrm{a}$ & $28.29 \mathrm{~A}$ \\
\hline CSSL B & $98.33 \mathrm{a}$ & $80.16 \mathrm{a}$ & $21.55 \mathrm{a}$ & $29.88 \mathrm{a}$ & $6.22 \mathrm{a}$ & $50.21 \mathrm{~B}$ \\
\hline CSSL C & $97.67 \mathrm{a}$ & $78.79 \mathrm{a}$ & $21.97 \mathrm{a}$ & $30.02 \mathrm{a}$ & $6.53 \mathrm{a}$ & $47.60 \mathrm{~B}$ \\
\hline CSSL D & $98.00 \mathrm{a}$ & $79.65 \mathrm{a}$ & $22.49 \mathrm{a}$ & $29.39 \mathrm{a}$ & $6.16 \mathrm{a}$ & $27.35 \mathrm{~A}$ \\
\hline CSSL E & $98.00 \mathrm{a}$ & $79.26 \mathrm{a}$ & $21.38 \mathrm{a}$ & $28.96 \mathrm{a}$ & $6.82 \mathrm{a}$ & $30.23 \mathrm{~A}$ \\
\hline CSSL F & $99.00 \mathrm{a}$ & $79.94 \mathrm{a}$ & $20.93 \mathrm{a}$ & $30.12 \mathrm{a}$ & $7.06 \mathrm{a}$ & $48.89 \mathrm{~B}$ \\
\hline CSSL G & $98.33 \mathrm{a}$ & $78.87 \mathrm{a}$ & $21.39 \mathrm{a}$ & $30.09 \mathrm{a}$ & $6.41 \mathrm{a}$ & $46.58 \mathrm{~B}$ \\
\hline CSSL H & $98.00 \mathrm{a}$ & $80.69 \mathrm{a}$ & $22.01 \mathrm{a}$ & $28.98 \mathrm{a}$ & $6.70 \mathrm{a}$ & $29.65 \mathrm{~A}$ \\
\hline CSSL I & $98.33 \mathrm{a}$ & $78.39 \mathrm{a}$ & $21.82 \mathrm{a}$ & $29.29 \mathrm{a}$ & $6.87 \mathrm{a}$ & $31.45 \mathrm{~A}$ \\
\hline CSSL K & $97.67 \mathrm{a}$ & $79.44 \mathrm{a}$ & $21.26 \mathrm{a}$ & $29.68 \mathrm{a}$ & $6.79 \mathrm{a}$ & $49.18 \mathrm{~B}$ \\
\hline
\end{tabular}

${ }^{\mathrm{z}} \mathrm{HD}$, heading date; PH, plant height; PL, panicle length; FLL, flag leaf length; FLW, flag leaf width; TN, tiller number; TA, tiller angle. Values with different lowercase letters or different capital letters indicate the difference on $5 \%(P<0.05)$ or $1 \%(P<0.01)$ levels of statistic significance, respectively, whereas values with the same letter indicate no statistical significance.

Table 3. Analysis of variance of sheath blight scores for 10 heterozygous chromosome segment substitution lines (hCSSLs) from each of two backcrosses

\begin{tabular}{|c|c|c|c|c|c|}
\hline Material and backcross & Variation source & Degree of freedom & Mean square & $F$ value & $P$ value \\
\hline \multicolumn{6}{|l|}{ hCSSLs } \\
\hline \multirow{3}{*}{ Jasmine85/Lemont } & Genotype & 9 & 10.5540 & 29.1970 & 0.0000 \\
\hline & Error & 406 & 0.3615 & $\ldots$ & $\ldots$ \\
\hline & Total & 415 & $\ldots$ & $\ldots$ & $\ldots$ \\
\hline \multirow[t]{3}{*}{ Minghui63/Lemont } & Genotype & 9 & 12.3467 & 19.4740 & 0.0000 \\
\hline & Error & 451 & 0.6340 & $\ldots$ & $\ldots$ \\
\hline & Total & 460 & $\ldots$ & $\ldots$ & $\cdots$ \\
\hline
\end{tabular}


2 ), and is almost consistent with that of $q S B-9^{J 85}-2$, whereas it has no overlap with those of $q S B-9^{J 85}-1, q S B-9^{M H 63}-1$, and $q S B-9^{M H 63}-2$. As a result, we validated the existence of $q S B-9^{J 85}-2$ and confirmed an SBR QTL on MH63, designated $q S B-9^{M H 63}-3$.

Because the resistant alleles of $q S B-9^{L M N T}$ came from the susceptible variety LMNT, the CSSLs that had the location of $q S B-9^{L M N T}$ replaced by a donor chromosomal segment should be more susceptible than LMNT. However, two such lines, CSSLs D and I, showed susceptibility similar to that of LMNT (Fig. 2; Table 6). Similarly, no statistically significant differences in the SB disease ratings among LMNT and hCSSLs D and I were found (Fig. 2; Table 4). These results indicate that, at least in the present study, $q S B-9^{L M N T}$ cannot be validated.

The resistance effects of $q S B-9^{J 85}-2$ and $q S B-9^{M H 63}-3$ to the SB disease. hCSSL E and CSSL E contained the smallest donor chromosomal segment, which included $q S B-9^{J 85}-2$ and $q S B-9^{M H 63}-3$, compared with the other lines and showed resistant phenotype. In addition, these two resistant lines shared the same TA with the susceptible control hCSSL A, CSSL A, and the recurrent parent LMNT. Therefore, we used these lines to estimate the resistance effects of $q S B-9^{J 85}-2$ and $q S B-9^{M H 63}-3$, and designated the two lines, CSSL-E- $q S B 9^{M H 63}$ and CSSL-E- $q S B 9^{J 85}$, as NILs of LMNT.

The results summarized in Table 7 indicate that $q S B-9^{J 85}-2$ and $q S B-9^{M H 63}-3$ reduced the $\mathrm{SB}$ disease severity score by 0.93 and 1.01 , respectively, in heterozygous condition in the 0-to-9 SB disease rating system. Furthermore, when the two SBR QTLs were in homozygous conditions, they were estimated to reduce the SB disease score by 1.10 for $q S B-9^{J 85}-2$ and 0.97 for $q S B-9^{M H 63}-3$. By comparing the effects of the SBR QTLs in heterozygous and homozygous conditions, we confirmed that $q S B-9^{J 85}-2$ and $q S B$ $9^{M H 63}-3$ both acted as dominant resistant genes. After taking into consideration variations in evaluation, we believe that both $q S B$ $9^{J 85}-2$ and $q S B-9^{M H 63}-3$ can reduce the SB disease score by approximately 1.0. Consequently, we conclude that $q S B-9^{J 85}-2$ and $q S B-9^{M H 63}-3$ have comparable resistance effects on SB resistance by reducing SB disease severity in a dominant heredity manner.

Allelic analysis among $q S B-9^{J 85}-2, q S B-9^{M H 63}-3$, and $q S B-9^{T Q}$. According to the integrated map (Fig. 1), we found that $q S B-9^{J 85}-2$, $q S B-9^{M H 63}-3$, and $q S B-9^{T Q}$ located in a very similar region. We then used three kinds of HLs, three NILs each containing one of the three SBR QTLs, and the LMNT control to test whether these SBR QTLs were controlled by the same allelic genes. The ANOVA and multi-comparisons results (Tables 8 and 9) showed that susceptible LMNT had a higher SB disease rating than that of the other six lines, reaching highly statistically significant difference $(P=$ 0.000 ); however, no statistically significant difference was found among lines $(P=0.346)$. This implied that three SBR QTLs had the same contribution to $\mathrm{SB}$ resistance, and no significantly stronger combination effects existed between any two of three SBR QTLs. According to the fact of dominant heredity of three SBR QTLs (Table 7) (30), we inferred that these SBR QTLs, from different donor parents, were controlled by the same allelic genes.

\section{Discussion}

Verification of SBR QTLs on the lower part of chromosome 9. In the present study, we developed two sets of CSSLs in a common japonica variety background that possessed chromosomal segments from two indica varieties on the long arm of chromosome 9. Based on the resistance performance of these CSSLs to the
SB disease, we found significant differences in what existed among these CSSLs, and that the CSSLs in each backcross could be divided into two classes: relatively resistant and susceptible (Table 4). Through determination of the genotype and phenotype of each CSSL, we confirmed the presence of $q S B-9^{M H 63}-3$ and $q S B-9^{J 85}-2$ and delimited them into a common region between markers Y83 and Y91.8, covering approximately $1,235 \mathrm{~kb}$ (Fig. 2). This common region is not consistent with the previous studies reported for $q S B$ $9^{M H 63}-1$, $q S B-9^{M H 63}-2$ (5), and $q S B-9^{J 85}-1$ (32) but it is almost identical to that reported by Liu et al. $(12,13)$ for $q S B-9^{J 85}-2$ (Fig. 1).

Several previous SBR QTL studies have reported that rice SB resistance was easily influenced by plant morphological and developmental changes, such as PH and $\operatorname{HD}(6,10,20,25,32,38)$. All rice lines in mapping populations used by Zou et al. (32) and Han et al. (5) were genetically stable, which was beneficial for phenotyping. The morphological and developmental traits, however, varied greatly among lines, and their influence on SB resistance could not be eliminated and, consequently, would have increased phenotyping errors. In addition, many other factors, such as population size variations and incomplete marker genotypes, that were commonly observed in preliminarily QTL-mapping studies could lead to deviations or inaccuracy in QTL interval determination $(9,11,26)$. The CSSLs we generated in this study were very similar to the recurrent parent LMNT in morphology and development (Table 2). Significant differences were only found in TA among CSSLs and among hCSSLs. Yu et al. (31) reported the isolation of QTL TAC1 controlling TA from the indica variety 'IR24' that was located in the integrated chromosome region covering the SBR QTLs tested in the present study (31). Therefore, we reasoned that the difference of lines in TA was attributed to the TAC1 gene and that the TQ or MH63 alleles in the TAC1 locus could result in bigger TAs. TA was also considered one of the plant characteristics that could affect SB disease development by altering plant microenvironment. However, in this study, we did not find significant effects on SB disease development derived from differences in TA, because no differences were found among CSSLs or hCSSLs with larger TA

Table 6. Multi-comparisons of sheath blight scores for Lemont and 10 chromosome segment substitution lines (CSSLs) from each of two backcrosses $^{\mathrm{z}}$

\begin{tabular}{lccccc}
\hline & \multicolumn{4}{c}{ CSSLs (2009) } \\
\cline { 2 - 3 } & \multicolumn{2}{c}{ Jasmine85/Lemont } & & \multicolumn{2}{c}{ Minghui63/Lemont } \\
\cline { 2 - 3 } \cline { 5 - 6 } Types & $\boldsymbol{N}$ & DS & & $\boldsymbol{N}$ & DS \\
\hline CSSL E & 50 & $6.54 \mathrm{~B}$ & & 50 & $6.52 \mathrm{~B}$ \\
CSSL C & 50 & $6.49 \mathrm{~B}$ & & 50 & $6.68 \mathrm{~B}$ \\
CSSL K & 50 & $6.62 \mathrm{~B}$ & & 50 & $6.45 \mathrm{~B}$ \\
CSSL G & 50 & $6.51 \mathrm{~B}$ & & 50 & $6.54 \mathrm{~B}$ \\
CSSL F & 50 & $6.81 \mathrm{~B}$ & & 50 & $6.62 \mathrm{~B}$ \\
CSSL D & 50 & $7.57 \mathrm{~A}$ & & 50 & $7.65 \mathrm{~A}$ \\
CSSL M & 50 & $7.71 \mathrm{~A}$ & & 50 & $7.70 \mathrm{~A}$ \\
CSSL H & 50 & $7.70 \mathrm{~A}$ & & 50 & $7.55 \mathrm{~A}$ \\
CSSL A & 50 & $7.64 \mathrm{~A}$ & & 50 & $7.49 \mathrm{~A}$ \\
CSSL B & 50 & $6.69 \mathrm{~B}$ & & 50 & $6.53 \mathrm{~B}$ \\
Lemont & 50 & $7.66 \mathrm{~A}$ & & 50 & $7.54 \mathrm{~A}$ \\
\hline
\end{tabular}

${ }^{\mathrm{z}} N=$ number of plants and DS $=$ disease scores. Values with different capital letters indicate the difference on $1 \%$ level of statistic significance $(P<0.01)$ while values with same letter indicate not statistically significant $(P>0.05)$.

Table 5. Analysis of variance of sheath blight scores for 10 chromosome segment substitution lines (CSSLs) from each of two backcrosses

\begin{tabular}{|c|c|c|c|c|c|}
\hline Material and backcross & Variation source & Degree of freedom & Mean square & $F$ value & $P$ value \\
\hline \multicolumn{6}{|l|}{ CSSLs } \\
\hline \multirow[t]{3}{*}{ Jasmine85/Lemont } & Genotype & 10 & 1.5777 & 30.019 & 0.0001 \\
\hline & Error & 44 & 0.0526 & $\ldots$ & $\ldots$ \\
\hline & Total & 54 & $\ldots$ & $\ldots$ & $\ldots$ \\
\hline \multirow[t]{3}{*}{ Minghui63/Lemont } & Genotype & 10 & 1.4259 & 27.753 & 0.0001 \\
\hline & Error & 44 & 0.0514 & $\ldots$ & $\ldots$ \\
\hline & Total & 54 & $\ldots$ & $\ldots$ & $\ldots$ \\
\hline
\end{tabular}


and normal TA in the presence of $q S B-9^{M H 63}-3$ or $q S B-9^{J 85}-2$ (Tables 4 and 6). Consequently, we believe that both $q S B-9^{M H 63}-3$ and $q S B-9^{J 85}-2$ are located in the common region between Y83 and Y91.8, where $q S B-9^{M H 63}-3$ was first identified.

However, for $q S B-9^{L M N T}$, derived from LMNT (12), the present study did not detect its measurable effect on SB resistance. All lines (hCSSLs D and I and CSSLs D and I) that harbored the introgressed LMNT segment supposedly containing the candidate $q S B$ $9^{L M N T}$ showed the same susceptible phenotype as the control lines (Tables 4 and 6). Interestingly, we also recorded one other SBR QTL, $q S B-11^{L E}$, that was reported in our previous studies and recently fine-mapped into a $74-\mathrm{kb}$ region $(33,34)$ but was not detected in the study by Pinson et al. (20) using the same cross. These discrepancies may be attributed to the differences in the rice stage used for inoculation, the fungal isolates used, and the different environments that occurred in the two studies. Isolate-specific and development-dependent QTLs have been reported in other disease systems $(19,21,36)$. In addition, Wang et al. (28) found that the resistance effects of $q S B-9^{T Q}$ and $q S B-12^{T Q}$ in the rice field at the adult stage were all smaller than those observed in a microchamber at the seedling stage. Therefore, there remains the possibility that the effect of $q S B-9^{L M N T}$ was too small to be measured in the rice field.

$q S B-9^{J 85}-2, q S B-9^{M H 63}-3$, and $q S B-9^{T Q}$ may represent the same SB resistance alleles. Two previous mapping regions for $q S B-9^{T Q}$ all covered the region between $\mathrm{Y} 83$ and $\mathrm{Y} 91.8$ containing $q S B-9^{M H 63}-3$ and $q S B-9^{J 85}-2$ (Fig. 1). Recently, a new moderately resistant line, MCR10277, was also detected to harbor an SBR QTL at the bottom of chromosome 9 (http://www.ricecap.uark.edu/ annual_reports.htm). Because the resistance of MCR10277 was believed to be from TQ, this SBR QTL on chromosome 9 was therefore inferred to be $q S B-9^{T Q}$ (http://www.ricecap.uark.edu/ annual_reports.htm). In the present study, we developed three HLs $\left(\mathrm{NIL}-q S B 9^{M H 63 / J 85}\right.$, NIL- $q S B 9^{M H 63 / T Q}$, and NIL- $q S B 9^{J 85 / T Q}$ ) to test whether $q S B-9^{M H 63}-3, q S B-9^{J 85}-2$, and $q S B-9^{T Q}$ were controlled by the same resistance alleles. Each of these lines contained a heterozygous genotype from two of three donor parents in the region containing the target SBR QTL. Theoretically, if not the same alleles control these SBR QTLs, their combination effects should be stronger than that of either alone because these SBR QTLs were all dominate resistance genes (Table 7) (30). However, we found no statistically significant differences in the SB scores between the three HLs and three NILs $(P=0.346)$, although they all showed higher resistance than that of the LMNT control $(P=0.000)$ (Tables 8 and 9). This indicated that the three SBR QTLs may represent the same alleles.

Furthermore, there are other characteristics of the three SBR QTLs that also support this conclusion. For instance, all three SBR QTLs acted as dominant resistant QTLs (Table 7) $(28,30)$. Furthermore, both $q S B-9^{M H 63}-3$ and $q S B-9^{J 85}-2$ showed similar contributions to SB resistance (Tables 7 and 9), reducing the SB disease score by approximately 1.0 in a 0 -to- 9 rating system. Although $q S B-9^{T Q}$ was found to reduce the SB disease score by 0.63 (28), 0.5 to 1.3 (37), and approximately 1.0 (30) in different studies, it showed an effect similar to that of $q S B-9^{M H 63}-3$ and $q S B-9^{J 85}-2$ in the present study (Table 9). Therefore, all three SBR QTLs were, in fact, quite similar in both their levels of resistance contribution and genetic modes. In addition, the resistant donor parents for the three SBR QTLs were from geographically proximal regions. For instance, MH63 and TQ were from southern China and J85 was from the Philippines. Therefore, it is likely that the origination of these varieties on SB resistance is the same, as well as the major SBR QTL on the long arm of chromosome 9.

We also noticed that, in the Wang study (28), $q S B-9^{T Q}$ was mapped into a small region that was only tagged by RM205 but not by RM245 (Fig. 1), which did not overlap with the region of $q S B$ $9^{J 85}-2$. This result was in contradiction to the above conclusion. We hypothesize that the real location for $q S B-9^{J 85}-2$ is a slight deviation from its confidence interval, because the confidence interval was just statistically inferred but not physically mapped. Fine mapping and isolation of the target SBR QTL should clarify this.

Currently, no SBR QTL has been isolated, and only one SBR QTL $\left(q S B-11^{L E}\right)$ has been fine-mapped by a map-based cloning strategy (33). In that study, a set of CSSLs with high mapping resolution in the candidate region of $q S B-11^{L E}$ was developed, and this was the critical step for the successful fine-mapping of $q S B$ $11^{L E}$. In the present study, we confirmed that the phenotypes from $q S B-9^{M H 63}-3$ and $q S B-9^{J 85}-2$ were measurable in the field using the CSSLs. However, the present CSSLs were apparently not enough to fine-map $q S B-9^{M H 63}-3$ and $q S B-9^{J 85}-2$ due to the low mapping resolution. The CSSL E, harboring the smallest introgressed segment, is the best line to use as the starting material to cross with the recurrent parent LMNT in order to develop a secondary set of CSSLs (or sub-CSSLs) with high resolution to fine-map this major SBR QTL.

Practical applications of the identified SBR QTLs. Developing cultivars with improved resistance to the SB disease has been

Table 9. Multi-comparisons of sheath blight scores for three chromosome segment substitution lines (CSSLs), three hybrid lines, and the Lemont control $^{\mathrm{z}}$

\begin{tabular}{lllc}
\hline Materials & $\boldsymbol{N}$ & DS & Difference \\
\hline NIL- $q S B 9^{T Q}$ & 50 & 6.06 & $\mathrm{~A}$ \\
NIL- $q S B 9^{M H 63 / T Q}$ & 50 & 6.13 & $\mathrm{~A}$ \\
NIL- $q S B 9^{185 / T Q}$ & 50 & 6.14 & $\mathrm{~A}$ \\
CSSL E- $q S B 9^{J 85}$ & 50 & 6.18 & $\mathrm{~A}$ \\
CSSL E- $q S B 9^{M H 63}$ & 50 & 6.20 & $\mathrm{~A}$ \\
NIL- $q S B 9^{M H 63 / J 85}$ & 50 & 6.22 & $\mathrm{~A}$ \\
Lemont & 50 & 7.08 & $\mathrm{~B}$ \\
\hline
\end{tabular}

${ }^{\mathrm{z}} N=$ number of plants, DS $=$ disease scores, and Difference $=$ difference in $1 \%$ significance level.

Table 7. Effects of $q S B-9^{J 85}$ and $q S B-9^{M H 63}$ on sheath blight (SB) resistance

\begin{tabular}{|c|c|c|c|c|c|c|c|}
\hline \multirow[b]{2}{*}{ QTL name } & \multirow[b]{2}{*}{ Crosses } & \multicolumn{2}{|c|}{ SB disease score } & \multirow[b]{2}{*}{ Heterozygous ${ }^{\mathrm{z}}$} & \multicolumn{2}{|c|}{ SB disease score } & \multirow[b]{2}{*}{ Homozygous } \\
\hline & & hCSSL A & hCSSL E & & CSSL A & CSSL E & \\
\hline$q S B-9^{J 85}$ & J85/LMNT & $8.13 \mathrm{~A}$ & $7.20 \mathrm{~B}$ & -0.93 & $7.64 \mathrm{~A}$ & $6.54 \mathrm{~B}$ & -1.10 \\
\hline$q S B-9 M H 63$ & MH63/LMNT & $8.15 \mathrm{~A}$ & $7.14 \mathrm{~B}$ & -1.01 & $7.49 \mathrm{~A}$ & $6.52 \mathrm{~B}$ & -0.97 \\
\hline
\end{tabular}

${ }^{\mathrm{y}} \mathrm{QTL}=$ quantitative trait loci.

${ }^{z}$ The effect was estimated by determining the SB disease score of heterozygous chromosome segment substitution line (hCSSL) E minus that of hCSSL A in the heterozygous condition (Heterozygous) and that of CSSL E minus CSSL A in the homozygous (Homozygous) condition. Values with the same letter indicate not statistically significant.

Table 8. Analysis of variance of sheath blight scores for three chromosome segment substitution lines, three hybrid lines, and the Lemont control

\begin{tabular}{lccccc}
\hline Materials & Variation source & Degree of freedom & Mean square & $\boldsymbol{F}$ value & 47.196 \\
\hline Seven lines & Genotype & 6 & 0.629 & & \\
& Error & 28 & 0.013 & & \\
& Total & 34 & & & \\
\hline
\end{tabular}


difficult using a traditional breeding strategy. The lack of major resistance genes in rice has made the utilization of SBR QTLs the only choice to improve rice resistance to the SB disease (38). $q S B$ $9^{M H 63}-3$ and $q S B-9^{J 85}-2$ showed a significant and stable effect on $\mathrm{SB}$ resistance. $q S B-9^{T Q}$ also has been confirmed to improve rice $\mathrm{SB}$ resistance, although $q S B-9^{M H 63}-3$ and $q S B-9^{J 85}-2$ can be used if no polymorphic markers exist between the $q S B-9^{T Q}$ donor parent and the varieties to be improved. Marchetti and Bollich (14) reported up to 5.1-fold relationship between yield loss and sheath blight rating. This means that a $5.1 \%$ reduction in yield loss can be expected by the introgression of either $q S B-9^{M H 63}-3$ or $q S B-9^{J 85}-2$ into LMNT-like varieties because they reduce SB disease severity by 1.0 degree (Tables 7 and 9). Zuo et al. (35) reported that $q S B$ $11^{L E}$ could rescue yield losses up to $10.71 \%$ in serious SB disease conditions (SB disease scores > 7.5), even though the SB disease severity score was only reduced by 0.74 degree. Beyond the direct benefit of being resistant to the SB fungus infection, the amount of fungal inoculum, which is directly proportional to the density of $R$. solani sclerotia in the field, may be reduced with continuous growth of rice cultivars with improved resistance using SBR QTLs. This may lead to a significant reduction in the overall costs spent on fungicide usage. Finally, using the verified DNA markers reported here, it is now possible to incorporate $q S B-9^{M H 63}-3$ and $q S B$ $9^{J 85}-2$ into commercial elite cultivars and improve their resistance to SB disease.

\section{Acknowledgments}

This work was partially supported by grants from the National Transgenic project (2012zx08009001, 2014ZX08001-003B), the Project Funded by the Priority Academic Program Development of Jiangsu Higher Education Institutions (PAPD), and the Jiangsu natural science foundation (SBK201320002). We thank M. Chern at the University of California-Davis for his helpful suggestions in language correction.

\section{Literature Cited}

1. Akshay, T., and Zhang, Q. G. 2003. Development of single segment substitution lines (SSSLs) and mapping of QTLs in rice (Oryza sativa L.). Mol. Plant Breed. 14:565-567.

2. Chen, Z. X., Zou, J. H., Tong, Y. H., Tang, S. Z., Wang, Z. B., Jiang, R. M., Ling, B., Tang, J., and Pan, X. B. 2000. A preliminary study on resources of resistance to rice sheath blight. Chin. J. Rice Sci. 14:15-18.

3. Eizenga, G. C., Prasad, B., Jackson, A. K., and Jia, M. H. 2013. Identification of rice sheath blight and blast quantitative trait loci in two different $O$. sativa/O. nivara advanced backcross populations. Mol. Breed. 31:889-907.

4. Fu, D., Chen, L., Yu, G. H., Liu, Y., Lou, Q. J., Mei, H. W., Xiong, L., Li, M. S., Xu, X. Y., and Luo, L. J. 2011. QTL mapping of sheath blight resistance in a deep-water rice cultivar. Euphytica 180:209-218.

5. Han, Y. P., Xing, Y. Z., Chen, Z. X., Gu, S. L., Pan, X. B., Chen, X. L., and Zhang, Q. F. 2002. Mapping QTLs for horizontal resistance to sheath blight in an elite rice restorer line, Minghui63. Acta Genet. Sin. 29:565-570.

6. Han, Y. P., Xing, Y. Y., Gu, S. L., Chen, Z. X., Pan, X. B., and Chen, X. L. 2003. Effect of morphological traits on sheath blight resistance in rice. Acta Bot. Sin. 45:825-831.

7. Ji, Q., Lu, J. F., Chao, Q., Gu, M. H., and Xu, M. L. 2005. Delimiting a rice wide-compatibility gene $\mathrm{s}_{5}{ }^{\mathrm{n}}$ to a $50 \mathrm{~Kb}$ region. Theor. Appl. Genet. 111:1495-1503.

8. Jia, L. M., Yan, W. G., Zhu, C. S., Agrama, H. A., Jackson, A., Yeater, K., Li, X. B., Huang, B. H., Hu, B. L., McClung, A., and Wu, D. X. 2012. Allelic analysis of sheath blight resistance with association mapping in rice. PLoS One 7:e32703. Online publication. doi:10.1371/journal.Pone.0032703

9. Kearsey, M. J., and Farquhar, A. G. L. 1998. QTL analysis in plants; where are we now? Heredity 80:137-142.

10. Li, Z. K., Pinson, S. R. M., Marshetti, M. A., Stansel, J. W., and Park, W. D. 1995. Characterization of quantitative trait loci (QTLs) in cultivated rice contributing to field resistance to sheath blight (Rhizoctonia solani). Theor. Appl. Genet. 91:374-381.

11. Liu, B. H. 2002. Statistical Genomics: Linkage, Mapping and QTL Analysis, 2nd ed. CRC Press, Boca Raton, FL.

12. Liu, G. J., Jia, Y. L., Correa-Victoria, F. J., Prado, G. A., Yeater, K. M., McClung, A. M., and Correll, J. C. 2009. Mapping quantitative trait loci responsible for resistance to rice sheath blight disease using greenhouse assays. Phytopathology 99:1078-1084.

13. Liu, G. J., Jia, Y. L., McClung, A., Oard, J.H., Lee, F.N., and Correll, J. C. 2013. Confirming QTLs and finding additional loci responsible for resistance to rice sheath blight disease. Plant Dis. 97:113-117.

14. Marchetti, M. A., and Bollich, C. N. 1991. Quantification of the relationship between sheath blight severity and yield loss in rice. Plant Dis. 75:773-775.
15. Murray, M. G., and Thompson, W. F. 1980. Rapid isolation of high molecular weight plant DNA. Nucleic Acids Res. 8:4321-4325.

16. Pan, X. B., Chen, Z. X., and Xu, J. Y. 1997. The effects of different methods of inoculation and Investigation on genetic research of resistance to rice sheath blight. J. Jiangsu Agric. Coll. 18:27-32.

17. Pan, X. B., and Rush, M. C. 1997. Studies in the U.S. on genetics and breeding of resistance to rice sheath blight. J. Jiangsu Agric. Coll. 18:57-63.

18. Pan, X. B., Zhang, Y. F., Zuo, S. M., and Chen, Z. X. 2005. Discussion on QTLs identification and application of important quantitative traits in crops. J. Yangzhou Univ. (Agric. Life Sci. Ed.). 26:50-55.

19. Perchepied, L., Dogimont, C., and Pitrat, M. 2005. Strain-specific and recessive QTLs involved in the control of partial resistance to Fusarium oxysporum f. sp. melonis race 1.2 in a recombinant inbred line population of melon. Theor. Appl. Genet. 111:65-74.

20. Pinson, S. R. M., Capdevielle, F. M., and Oard, J. H. 2005. Confirming QTLs and finding additional loci conditioning sheath blight resistance in rice using recombinant inbred lines. Crop Sci. 45:503-510.

21. Poland, J. A., Balint-Kurti, P. J., Wisser, R. J., Pratt, R. C., and Nelson, R. J. 2009. Shades of gray: the world of quantitative disease resistance. Trends Plant Sci. 14:21-29.

22. Rush, M. C., Hoff, B. J., and Mcllrath, W. O. 1976. A uniform disease rating system for rice disease in the United States. Page 64 in: Proc. 16th Rice Tech. Working Group, Lake Charles, LA.

23. Salvi, S., and Tuberosa, R. 2005. To clone or not to clone plant QTLs: present and future challenges. Trends Plant Sci. 10:297-304.

24. Sato, H., Ideta, O., Audo, I., Kunihiro, Y., Hirabayashi, H., Iwano, M., Miyasaka, A., Nemoto, H., and Imbe, T. 2004. Mapping QTLs for sheath blight resistance in the rice line WSS2. Breed. Sci. 54:265-271.

25. Singh, A., Rohilla, R., Singh, U. S., Savary, S., Willocquet, L., and Duvelller, E. 2002. An improved inoculation technique for sheath blight of rice caused by Rhizoctonia solani. Can. J. Plant Pathol. 24:65-68.

26. Srinivasachary, Willocquet, L., and Savary, S. 2011. Resistance to rice sheath blight (Rhizoctonia solani Kühn) [(teleomorph: Thanatephorus cucumeris (A. B. Frank) Donk.) disease: current status and perspectives. Euphytica 178:1-22.

27. Tan, C. X., Ji, X. M., Yang, Y., Pan, X. B., Zuo, S. M., Zhang, Y. F., Zou, J. H., Chen, Z. X., Zhu, L. H., and Pan, X. B. 2005. Identification and marker assisted selection of two major quantitative genes controlling rice sheath blight resistance in backcross generations. Acta Genet. Sin. 32:399-405.

28. Wang, Y., Pinson, S. R. M., Fjellstrom, R. G., and Tabien, R. E. 2012. Phenotypic gain from introgression of two QTL, $q S B 9-2$ and $q S B 12-1$, for rice sheath blight resistance. Mol. Breed. 30:293-303.

29. Xie, X. W., Xu, M. R., Zang, J. P., Sun, Y., Zhu, L. H., Xu, J. L., Zhou, Y. L., and Li, Z. K. 2008. Genetic background and environmental effects on expression of QTL for sheath blight resistance in reciprocal introgression lines of rice. Acta Agron. Sin. 34:1885-1893.

30. Yin, Y. J., Zuo, S. M., Wang, H., Chen, Z. X., Gu, S. L., Zhang, Y. F., and Pan, X. B. 2009. Evaluation of the effect of $q S B-9(T q)$ involved in quantitative resistance to rice sheath blight using near-isogenic lines. Can. J. Plant Sci. 4:731-737.

31. Yu, B. S., Lin, Z. W., Li, H. X., Li, X. J., Li, J. Y., Wang, Y. H., Zhang, X., Zhu, Z. F., Zhai, W. X., Wang, X. K., Xie, D. X., and Sun, C. Q. 2007. TAC1, a major quantitative trait locus controlling tiller angle in rice. Plant $J$. 52:891-898.

32. Zou, J. H., Pan, X. B., Chen, Z. X., Xu, J. Y., Lu, J. F., Zhai, W. X., and Zhu, L. H. 2000. Mapping quantitative trait loci controlling sheath blight resistance in two rice cultivars (Oryza sativa L.). Theor. Appl. Genet. 101:569-575.

33. Zuo, S. M., Yin, Y. J., Pan, C. H., Chen, Z. X., Zhang, Y. F., Gu, S. L., Zhu, L. H., and Pan, X. B. 2013. Fine mapping of $q S B-11^{L E}$, the QTL that confers partial resistance to rice sheath blight. Theor. Appl. Genet. 126:1257 1272.

34. Zuo, S. M., Yin, Y. J., Zhang, L., Zhang, Y. F., Chen, Z. X., Gu, S. L., Zhu, L. H., and Pan, X. B. 2011. Effect and breeding potential of $q S B-11^{L E}$, a sheath blight resistance quantitative trait loci from a susceptible rice cultivar. Can J Plant Sci. 91:191-198.

35. Zuo, S. M., Yin, Y. J., Zhang, L., Zhang, Y. F., Chen, Z. X., and Pan, X. B. 2007. Breeding value and further mapping of a QTLqSB-11 conferring the rice sheath blight resistance. Chin. J. Rice Sci. 21:136-142.

36. Zuo, S. M., Yin, Y. J., Zhang, Y. F., Chen, Z. X., and Pan, X. B. 2006. Progress on cloning of quantitative disease resistance gene in plant and studying its possible resistance mechanism. Mol. Plant Breed. 4:603-613.

37. Zuo, S. M., Zhang, L., Wang, H., Yin, Y. J., Zhang, Y. F., Chen, Z. X., Ma, Y. Y., and Pan, X. B. 2008. Prospect of the QTL- $q S B-9^{T Q}$ utilized in molecular breeding program of japonica rice against sheath blight. J. Genet. Genomics 35:499-505.

38. Zuo, S. M., Zhang, Y. F., Chen, Z. X., Chen, X. J., and Pan, X. B. 2010. Current progress on genetics and breeding in resistance to rice sheath blight. Sci. Sin Vitae 40:1014-1023.

39. Zuo, S. M., Zhang, Y. F., Yin, Y. J., Chen, Z. X., and Pan, X. B. 2006. Establishment and improvement of inoculation technique and rating system in researching rice sheath blight resistance in field. J. Yangzhou Univ. (Agric. Life Sci. Ed.). 27:57-61 